\section{Possible Four-Electrode Configurations for Measuring Cardiac Tissue Fiber Rotation}

\author{
Barbara M. Johnston and Peter R. Johnston*
}

\begin{abstract}
A number of electrode configurations, based on the usual fourelectrode probe, are analysed in relation to the effect that changes in cardiac fiber rotation have on the potentials measured. Simulations are carried out using a mathematical model and a new solution technique, based on Fourier series followed by a simple one-dimensional finite difference scheme. This electrode analysis leads to the proposal of an in-principle method for determining cardiac fiber rotation.
\end{abstract}

Index Terms-Bidomain model, electrodes, electromagnetic field simulation, fiber rotation.

\section{INTRODUCTION}

Cardiac tissue is electrically anisotropic because current is able to flow more easily in one direction than the other. This anisotropy occurs in two ways: within each 2-D sheet, cell geometry and gap junctions determine a preferred direction; and, when these sheets are stacked to form a 3-D structure, the orientation of the preferred direction often rotates from one layer to the next. The importance of obtaining accurate measurements, for the cardiac electrical conductivities and total fiber rotation through the cardiac tissue, has been reflected in a number of experimental and modelling studies. These show that including cardiac tissue anisotropy is essential to produce realistic cardiac and thoracic potential distributions. In particular, fiber rotation has been shown to affect the predictive value of models of defibrillation, wavefront propagation and the initiation and maintenance of re-entry [1]-[3].

Cardiac fiber rotation has usually been found using difficult and time-consuming techniques such as slicing away the fibers layer by layer [4] or from detailed morphological studies [5]. Fiber orientation can also be estimated by curve fitting epicardial stimulus isopotential and activation isochrones, both during and after pace mapping [3]. Another technique involves magnetic resonance imaging (MRI) diffusion tensor imaging, which exploits the dependence of the molecular diffusion of water on the tissue microstructure [6].

The four-electrode technique [7] is the basis of a number of different electrode systems that have been proposed for the measurement of cardiac electrical conductivities [8]-[12]. They all consist of a probe containing four equi-spaced electrodes, where subthreshold current is applied via the outer electrodes and the potential is measured at the inner electrodes. In addition to experimental measurements, a tissue model must be adopted to interpret these results. This is now generally accepted to be a bidomain model [13], rather than the monodomain model used in two of the above studies [8], [9].

This paper makes use of a recently presented [14] mathematical bidomain model, which is able to include the effects of fiber rotation. The model, solved using Fourier series and a simple one-dimensional finite difference scheme, is used to study the effect of varying fiber rotation on the potentials measured, for various four-electrode configurations. The relationships between the potential measured and the change

Manuscript received May 15, 2006; revised August 5, 2006. This work was supported in part by the Australian Research Council. Asterisk indicates corresponding author.

B. M. Johnston is with the School of Science, Griffith University, Nathan 4111, Queensland, Australia (e-mail: Barbara.Johnston@ griffith.ed.u.au).

*P. R. Johnston is with the School of Science, Griffith University, Nathan 4111, Queensland, Australia (e-mail: P.Johnston@griffith.edu.au).

Digital Object Identifier 10.1109/TBME.2006.890511 in total cardiac fiber rotation angle, for certain of these electrodes, leads to the proposal of an in-principle method for determining cardiac tissue fiber rotation. This paper will look systematically at a number of generalisations of the usual four-electrode configuration, including some arrangements which are not co-linear, to assess their suitability for use with this method.

\section{METHODS}

In this work, the cardiac tissue is described by a bidomain model [13] which is able to include the effects of cardiac fiber rotation. Assuming that the conductivities in the two directions perpendicular to the fibers ( $y$ and $z$ ) are the same, four conductivity values, $g_{i l}, g_{i t}, g_{e l}$, and $g_{e t}$ are necessary to account for differences in conductivity in the intracellular $(i)$ and extracellular $(e)$ domains and in the longitudinal $(l)$ and transverse $(t)$ directions relative to the fibers. Equations for the intracellular and extracellular potentials in the tissue and the electric potential in the blood are then expressed in terms of these conductivity values as well as conductivity tensors, which will be described below.

To describe the fiber rotation, it is assumed that: 1) the cardiac fiber layers are parallel to the epicardium [4]; 2) the fibers are aligned with the positive $x$-axis at the epicardium; 3 ) the fiber rotation direction rotates anticlockwise from the epicardium to the endocardium; 4) that this rotation varies linearly with depth [2]. These assumptions lead to conductivity tensors, whose terms include combinations of the conductivity values and $\cos \alpha z$ or $\sin \alpha z$, where $\alpha$, the total fiber rotation angle through the tissue, lies between $0^{\circ}$ and $180^{\circ}$ [5]. Full details of the model can be found in [14].

The boundary conditions used to solve the model are based on the following assumptions [15] (and references therein): 1) the epicardium is insulated; 2) there is continuity of extracellular potential and current, across the interface between the tissue and the blood; 3) the intracellular space is insulated by the extracellular space.

A Fourier Series approach, used to solve the bidomain equations for the electric potential, subject to the boundary conditions, results in two sets of four ordinary differential equations, which are solved numerically by applying a one dimensional finite difference scheme. This gives a banded system of linear algebraic equations that are solved using standard techniques [16]. Summation of the series then yields the potentials. Full details of the solution method can be found in [14], including a full list of the modelling parameters used. These include nominal conductivity values of $g_{i l}=g_{e l}=2.6 \times 10^{-3} \mathrm{~S} / \mathrm{cm}, g_{i t}=$ $2.6 \times 10^{-4} \mathrm{~S} / \mathrm{cm}$ and $g_{\text {et }}=2.6 \times 10^{-3} \mathrm{~S} / \mathrm{cm}$.

The four-electrode configurations, which will be considered in this paper, are shown in Fig. 1(a)-(f), where the current injection electrodes are indicated by + and - and the measurement electrodes by $\bullet$. For each electrode configuration described below, two cases of electrode spacing are considered: a "close" case using $250 \mu \mathrm{m}$ and a "wide" case using $1 \mathrm{~mm}$. The six configurations are: 1) Surface Probe [7]; 2) Vertical Probe [8]; 3$)$ Angled Probes $\left(\theta_{37}\right.$ Probe, $\theta_{53}$ Probe and $\theta_{66}$ Probe), which correspond to insertion angles $\theta=\arctan (3 / 4) \approx 37^{\circ}$, $\theta=\arctan (4 / 3) \approx 53^{\circ}$ and $\theta=\arccos (2 / 5) \approx 66^{\circ}$, respectively; 4) Two Probes; 5) Three Probes and 6) Four Probes. These electrode configurations are then studied to determine the effect that varying the total fiber rotation angle $\alpha$ has on the potentials measured.

In addition, a study is conducted to attempt to determine the sensitivity of the potentials to changes in the values of the conductivities. This involves increasing all four nominal conductivity values by $1 \%$, then decreasing them all by $1 \%$ and repeating these steps using $5 \%$. Plots of potential difference versus total fiber rotation angle are then produced, so that the potentials can be compared with the original values. 


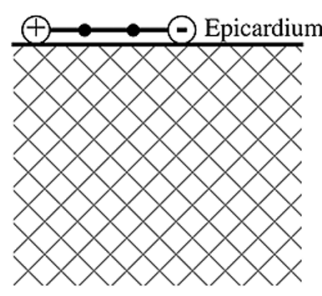

(a) Surface

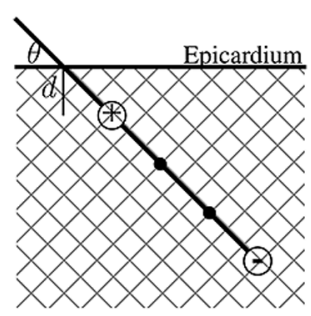

(c) Angled

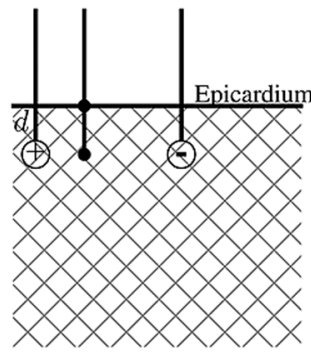

(e) Three Probes

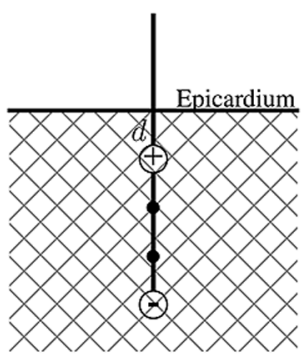

(b) Vertical

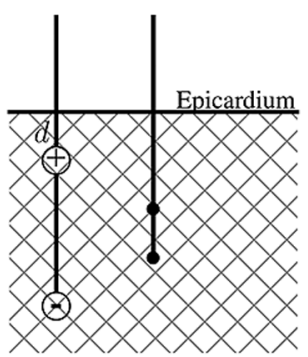

(d) Two Probes

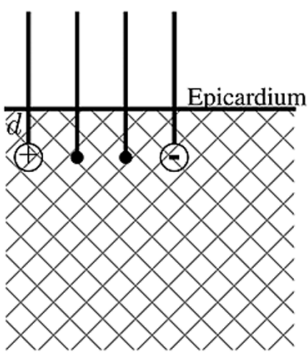

(f) Four Probes
Fig. 1. Schematic diagram for the various probe configurations. Current source and sink are denoted by + and - , respectively, with $\bullet$ for the measuring electrodes. Diagrams are not to scale.

\section{RESULTS}

The potential difference is calculated at the measuring electrodes, for a series of total fiber rotation angles $\alpha$ and at various depths $d$, as shown in Fig. 1, for the "close" and then "wide" electrode configurations. The results show two different qualitative behaviours. The first is shown by the surface horizontal probe and the single vertical probe. For these probes, the potential difference measured is not affected by changes in the degree of fiber rotation nor is it affected by how deep the probe is placed into the tissue (vertical probe). These results hold for both the "close" and "wide" electrode spacings. The remainder of the probes show a second type of behaviour, where changes in the degree of fiber rotation and depth of probe placement do affect the potentials measured. An example of these effects is given in Fig. 2, which shows the potential difference measured as a function of the total fiber rotation angle $\alpha$, for various depths $d$, in this case for the four probe configuration Fig. 1(f).

The graph contains two distinct types of curves: those that are monotonically decreasing with increasing degree of the total fiber rotation angle $(d \leq 0.5 \mathrm{~cm})$ and those which initially decrease, then reach a minimum and then increase again $(d>0.5 \mathrm{~cm})$. For the monotonic curves, it can be seen that the drop in voltage, between $0^{\circ}$ and $180^{\circ}$ of the total fiber rotation angle, increases as the electrodes are inserted deeper into the the tissue. These plots are typical of those which were found for any of the electrode configurations of the second type, except

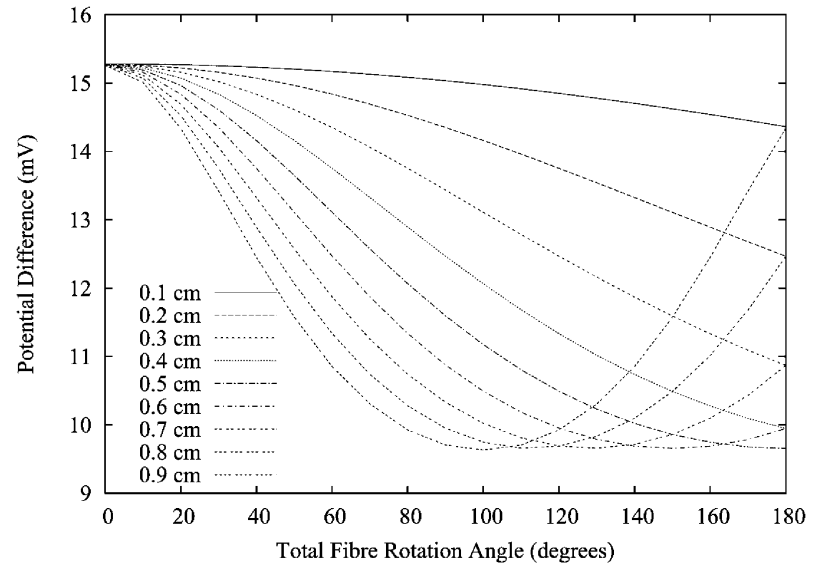

Fig. 2. Potential differences measured, using the four probe configuration, for varying degrees of the total fiber rotation angle and depth of the current injection electrode.

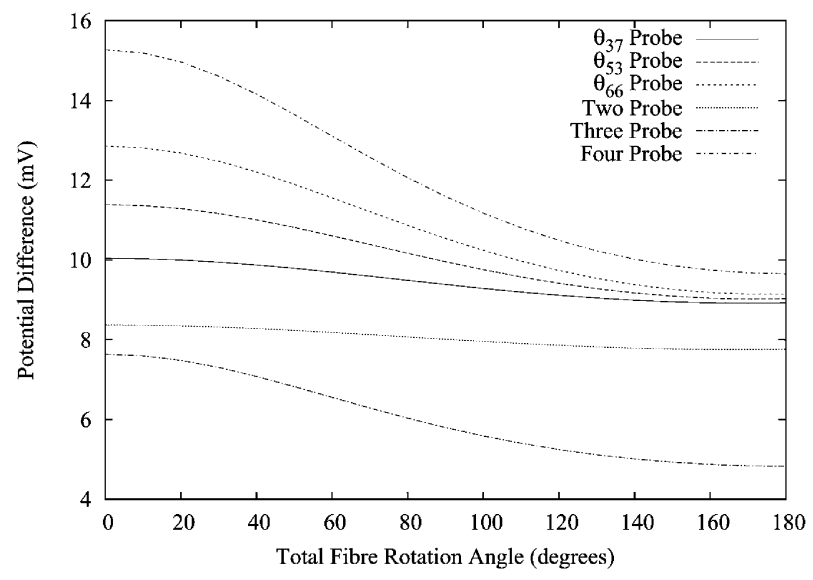

Fig. 3. Potential differences measured, for varying degrees of the total fiber rotation angle, using the various electrode configurations. The (top) current injection electrode is placed $0.5 \mathrm{~cm}$ below the epicardium.

for the magnitude of the voltages measured. For example, the "wide" electrode spacing has voltages of around one-quarter of the "close" case values.

A second set of similar plots, where $d=0.5 \mathrm{~cm}$ only, is shown in Fig. 3, so that a comparison between the different electrode configurations can be made. It can be seen that the measured voltage depends on the probe configuration chosen, but that, in all cases, the measured voltage decreases with increasing total fiber rotation angle. The four probe configuration gives the highest measured voltages.

A different set of plots in Fig. 4 shows the drop in potential between $0^{\circ}$ and $180^{\circ}$, as a percentage of the initial potential difference. It can be seen for all electrode configurations that the relative potential drop increases as the depth increases (up to $d=0.5 \mathrm{~cm}$ ). The plot for the three probe configuration is superimposed on the four probe configuration and these two show the largest relative potential drop at all depths.

The final plot, given in Fig. 5, shows the sensitivity of the potentials to $1 \%$ and $5 \%$ changes in the conductivity values, for the four probe electrode arrangement of Fig. 1(f), where $d=0.5 \mathrm{~cm}$. From this it can be seen that, over the bulk of the graph, a variation of $1 \%$ in the conductivities leads to a variation in the total fiber rotation angle of around $\pm 3^{\circ}$, while a variation of $5 \%$ in the conductivities changes the total fiber rotation angle by less than $\pm 15^{\circ}$. 


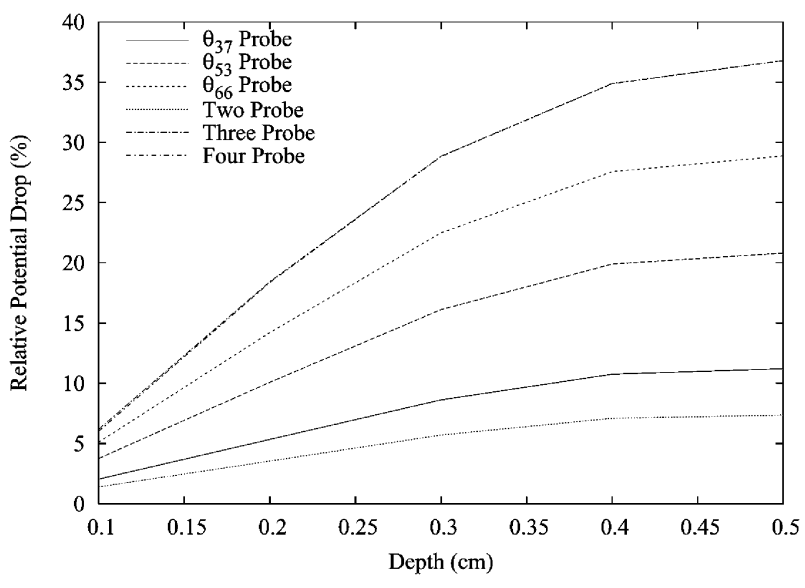

Fig. 4. Relative Potential Drop across the range of total fiber rotation angles, for the various electrode configurations, with the (top) current injection electrode placed at depths between 0.1 and $0.5 \mathrm{~cm}$.

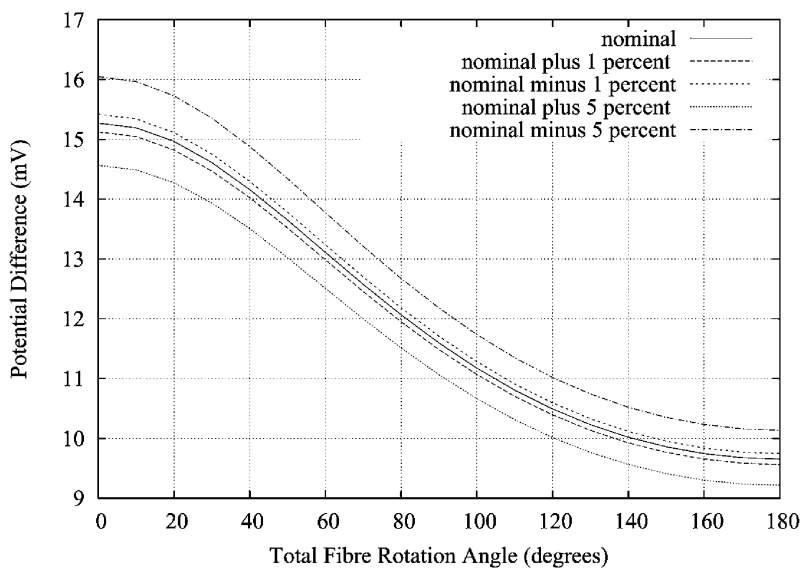

Fig. 5. Potential differences measured, for varying degrees of the total fiber rotation angle, using the four electrode configuration. The current injection electrode is placed $0.5 \mathrm{~cm}$ below the epicardium. The potential is calculated for all four nominal conductivities $+1 \%$ and then $-1 \%$; or $+5 \%$ and then $-5 \%$, as indicated.

\section{DISCUSSION}

The purpose of this study was to simulate various electrode configurations to determine their suitability for use with a method for finding total cardiac fiber rotation through the tissue. It has been demonstrated that a number of the electrode configurations [Fig. 1(c)-(f)] produce a qualitatively similar set of curves (Fig. 2), when potential measured is plotted against total fiber rotation angle, for various depths of the (top) current electrode. This relationship can be then be used to determine the total fiber rotation angle, as detailed below.

Once one of the electrode configurations [Fig. 1(c)-(f)] has been constructed, in principle all that is required is that the voltage is found at the measuring electrodes. This measurement would then be used with a plot similar to that in Fig. 2, for the particular electrode configuration and value of $d$ used, and a unique value for fiber rotation angle through the whole tissue could then be read off the plot. Note that a depth $d$ of $0.5 \mathrm{~cm}$ or less should be used so that the potential difference curve (Fig. 2) is monotonic.

In practice, there are a number of points which require consideration, including difficulties associated with making the experimental measurements, which will not be addressed here. Others include the difficulty of constructing such small, closely spaced electrodes and the fact that it is assumed that the bidomain electrical conductivities are already known.

The method presented here for finding fiber rotation is based on the four-electrode technique. Although the effect of electrode size and spacing, on resistivity measurements in skeletal muscle, has been studied by Wang et al. [17], few measurements [8]-[10] of conductivities in cardiac tissue have actually been made using the four-electrode technique, due to the difficulties associated with electrodes and electrode spacings of micrometre order. However, considerable recent interest has been shown in this field [11], [12], [18] and a number of studies, which demonstrate the feasibility of making various types of cardiac measurements using small electrodes with close spacings, have been conducted. These include studies by Witkowski et al. [19], who used five-element electrode arrays containing $25 \mu \mathrm{m}$-diameter silver electrodes with a center-to-center spacing of $65 \mu \mathrm{m}$ and Kim et al. [20], who used a micromachined four-shank probe, with center-to-center shank spacings of $750 \mu \mathrm{m}$, where each shank contained an array of three electrodes at different levels, $300 \mu \mathrm{m}$ apart.

Therefore, it may well be possible in the future, to determine the cardiac conductivities much more accurately than those currently available [10], [21]-[23], which vary considerably due to different experimental arrangements and modelling assumptions. The experimental errors quoted by Clerc [21] are around 8\%, except for $g_{\text {et }}$, while those of Roberts and Sher [23] are larger. The sensitivity analysis for the proposed method indicates that errors in the conductivity of $\pm 5 \%$ would lead to errors in the total fiber rotation angle of less than $\pm 15^{\circ}$, except near the epicardium or the endocardium. As a comparison, studies, which measure fiber rotation in the left ventricle, have found errors of around $12 \%$ [3] for the pace mapping method and $20 \%$ [6] and $40 \%$ [3] for the MRI method.

\section{CONCLUSION}

Four different electrode configurations, suitable for measuring the total cardiac tissue fiber rotation angle, are presented here. All are based on the usual four-electrode arrangement [7], except that the electrodes are introduced vertically into the myocardium on either two, three or four probes, or at an angle on a single probe. The mathematical model and solution technique described here are used, for a particular electrode configuration, to produce plots of potential measured, for a range of fiber rotation angles. These plots could then be used in conjunction with actual measurements of potential to determine the total fiber rotation angle throughout the cardiac tissue.

Decisions regarding which electrode configuration may be the most suitable for finding the total fiber rotation angle need to take into account a number of factors: firstly, "close" electrode spacings lead to higher voltage readings; secondly, both the magnitude of the voltage measured and the drop in voltage across the range of total fiber rotation angles are important in discriminating between voltage readings and lastly, the practical considerations involved in constructing the electrodes and measuring the potentials.

\section{REFERENCES}

[1] A. E. Pollard, N. Hooke, and C. S. Henriquez, "Cardiac propagation simulation," Crit. Rev. Biomed. Eng, vol. 20, pp. 171-210, 1992.

[2] P. Colli-Franzone and L. Guerri, "Spreading of excitation in 3-D models of the anisotropic cardiac tissue I: Validation of the eikonal model," Math. Biosci., vol. 113, pp. 145-209, 1993.

[3] A. L. Muzikant, E. W. Hsu, P. D. Wolf, and C. S. Henriquez, "Region specific modeling of cardiac muscle: Comparison of simulated and experimental potentials," Ann. Biomed. Eng., vol. 30, pp. 867-883, 2002.

[4] I. J. LeGrice, P. J. Hunter, and B. H. Smail, "Laminar structure of the heart: A mathematical model," Am. J. Physiol., vol. 272, pp. H2466-H2476, 1997. 
[5] D. D. Streeter, "Gross morphology and fiber geometry of the heart," in Handbook of Physiology, R. M. Berne, Ed. Baltimore, MD: Williams \& Williams, 1979, vol. 1, ch. 2: The Cardiovascular System, pp. 61-112.

[6] P. A. Helm, H.-J. Tseng, L. Younes, E. R. McVeigh, and R. L. Winslow, "Ex vivo 3D diffusion tensor imaging and quantification of cardiac laminar structure," Magn. Reson. Med., vol. 54, pp. 850-859, 2005.

[7] R. Plonsey and R. C. Barr, "The four-electrode resistivity technique as applied to cardiac muscle," IEEE Trans. Biomed. Eng., vol. BME-29, pp. 541-546, 1982.

[8] A. van Oosterom, R. W. de Boer, and R. T. Van Dam, "Intramural resistivity of cardiac tissue," Med. Biol. Eng. Comput., vol. 17, pp. 337-343, 1979.

[9] P. Steendijk, G. Mur, E. T. van der Velde, and J. Baan, "The four-electrode resistivity technique in anisotropic media: Theoretical analysis and application on myocardial tissue in vivo," IEEE Trans. Biomed. Eng., vol. 40, no. 11, pp. 1138-1147, Nov. 1993.

[10] P. Le Guyader, P. Savard, and F. Trelles, "Measurement of myocardial conductivities with an eight-electrode technique in the frequency domain," in Proc. 17th IEEE-EMBS, 1995, pp. 71-72.

[11] P. Le Guyader, F. Trelles, and P. Savard, "Extracellular measurement of anisotropic bidomain myocardial conductivities. I. Theoretical analysis," Ann. Biomed. Eng., vol. 29, pp. 862-877, 2001.

[12] R. C. Barr and R. Plonsey, "Electrode systems for measuring cardiac impedances using optical transmembrane potential sensors and interstitial electrodes-Theoretical design," IEEE Trans. Biomed. Eng., vol. 50, no. 8, pp. 925-934, Aug. 2003.

[13] L. Tung, "A bi-domain model for describing ischaemic myocardial DC potentials," Ph.D. dissertation, Massachusetts Inst. Technol., Cambridge, 1978.

[14] B. M. Johnston, P. R. Johnston, and D. Kilpatrick, "A new approach to the determinination of cardiac potential distributions: Application to the analysis of electrode configurations," Math. Biosci., vol. 202, no. 2, pp. 288-309, 2006.

[15] R. M. Gulrajani, Bioelectricity and Biomagnetism. New York: Wiley, 1998.

[16] H. P. William, P. F. Brian, A. T. Saul, and T. V. William, Numerical Recipes, The Art of Scientific Computing, 2nd ed. Cambridge, MA: Cambridge Univ. Press, 1992.

[17] Y. Wang, P. H. Schimpf, D. R. Haynor, and Y. Kim, "Geometric effects on resistivity measurements with four- electrode probes in isotropic and anisotropic tissues," IEEE Trans. Biomed. Eng., vol. 45, no. 7, pp. 8-19, Jul. 1998.

[18] A. E. Pollard and R. C. Barr, "Cardiac micro-impedance measurement in two-dimensional models using multisite interstitial stimulation," Am. J. Physiol.-Heart Circulatory Physiol., vol. 290, no. 5, pp. H1976-H1987, 2006.

[19] F. X. Witkowski, K. M. Kavanagh, P. A. Penkoske, and R. Plonsey, "In vivo estimation of cardiac transmembane current," Circ. Res., vol. 72, pp. 424-439, 1993.

[20] C.-S. Kim, S. Ufer, C. M. Seagle, C. L. Engle, H. T. Nagle, T. A. Johnson, and W. E. Cascio, "Use of micromachined probes for the recording of cardiac electrograms in isolated heart tissues," Biosensors Bioelectron., vol. 19, no. 9, pp. 1109-1116, Apr. 2004.

[21] L. Clerc, "Directional differences of impulse spread in trabecular muscle from mammalian heart," J. Physiol., vol. 255, pp. 335-346, 1976.

[22] D. E. Roberts, L. T. Hersh, and A. M. Scher, "Influence of cardiac fiber orientation on wavefront voltage, conduction velocity and tissue resistivity in the dog," Circ. Res., vol. 44, pp. 701-712, 1979.

[23] D. E. Roberts and A. M. Scher, "Effects of tissue anisotropy on extracellular potential fields in canine myocardium in situ," Circ. Res., vol. 50, pp. 342-351, 1982.

\section{Study of On-Line Adaptive Discriminant Analysis for EEG-Based Brain Computer Interfaces}

\author{
C. Vidaurre*, A. Schlögl, R. Cabeza, R. Scherer, and \\ G. Pfurtscheller
}

\begin{abstract}
A study of different on-line adaptive classifiers, using various feature types is presented. Motor imagery brain computer interface (BCI) experiments were carried out with 18 naive able-bodied subjects. Experiments were done with three two-class, cue-based, electroencephalogram (EEG)-based systems. Two continuously adaptive classifiers were tested: adaptive quadratic and linear discriminant analysis. Three feature types were analyzed, adaptive autoregressive parameters, logarithmic band power estimates and the concatenation of both. Results show that all systems are stable and that the concatenation of features with continuously adaptive linear discriminant analysis classifier is the best choice of all. Also, a comparison of the latter with a discontinuously updated linear discriminant analysis, carried out in on-line experiments with six subjects, showed that on-line adaptation performed significantly better than a discontinuous update. Finally a static subject-specific baseline was also provided and used to compare performance measurements of both types of adaptation.
\end{abstract}

Index Terms-AAR, automatic adaptive classification, band power estimates, BCI, Kalman filtering, LDA, on-line adaptation, QDA.

\section{INTRODUCTION}

An electroencephalogram (EEG)-based brain computer interface (BCI) is a system which enables people to control devices using signals recorded from the scalp. One of the goals of such a device is to assist patients who have highly compromised motor functions, such as completely paralyzed patients with e.g., amyotrophic lateral sclerosis [1]-[11].

A BCI is divided in different modules: preprocessing, feature extraction, classification and feedback. Various signals are used in BCI systems, but our experiences were based in EEG signals, which can vary in time. Therefore, adaptation of modules like feature extraction or/and classification is a very important issue in BCI research. Regarding the classification module, up to now classifiers were manual and discontinuously updated after a number of runs, and the moment when to perform this upgrade was decided by the researcher depending on subjective factors such as his/her experience, [4], [12], [13]. Because of the disadvantage of this manual adaptation, recently several BCI research groups have started to work on automatic adaptation of the classifiers [14]-[18].

Our group presents in this paper results from on-line experiments with two different automatic adaptive classifiers and three different feature extraction techniques. The adaptation of the classifier is done in a

Manuscript received November 17, 2005; revised August 11, 2006. This work was supported in part by the Spanish Ministry of Culture and Education under Grant AP-2000-4673, in part by the L. B. Gesellschaft, Austria, and in part by "Fonds zur Förderung der wissenschaftlichen Forschung", Austria, under Project 16326-BO2. Asterisk indicates corresponding author.

*C. Vidaurre is with the Department of Electrical and Electronic Engineering, Public University of Navarre, Campus Arrosadia s/n, 31006 Pamplona, Spain (e-mail: carmen.vidaurre@unavarra.es).

A. Schlögl is with the Human-Computer Interface Department, Graz University of Technology, 8010 Graz, Austria.

R. Cabeza is with the Department of Electrical and Electronic Engineering, Public University of Navarre, 31006 Pamplona, Spain.

R. Scherer and G. Pfurtscheller are with the BCI-Lab Computer Graphics Department, Graz University of Technology, $8010 \mathrm{Graz}$, Austria.

Color versions of Figs. 4-6 are available online at http://ieeexplore.ieee.org.

Digital Object Identifier 10.1109/TBME.2006.888836 